\title{
Typical Evolution of a Cutaneous Anthrax Lesion
}

\author{
Cansu Çimen \\ Infectious Diseases and Clinical Microbiology Clinic, Ardahan State Hospital, Ardahan, Turkey
}

\begin{abstract}
An illustrated case of cutaneous anthrax acquired in eastern Turkey was described in this report. A 56-year-old female patient applied to the infectious diseases outpatient clinic with a painless, dark-colored swelling over her right middle finger accompanied by edema extending to the right hand. Typical disease course of a cutaneous anthrax lesion evolved in a few days. This case report was presented in order to help clinicians to recognize the different stages of the disease in clinical practice.

Keywords: anthrax , cutaneous anthrax, eschar, edema
\end{abstract}

A nthrax is a zoonotic infection mainly affecting herbivores and caused by Bacillus anthracis. Humans can contract the disease after direct or indirect expo1 sure to animals or animal products (1). Human-to -human transmission has never been reported (2). Worldwide, most of the cases are among persons who come in contact with animals in agricultural regions of south and central America, sub-Saharan Africa, central and southwestern Asia, and southern and eastern Europe $(3,4)$. Besides, anthrax is still an endemic disease in Turkey (5). Depending on the inoculation site; cutaneous, gastrointestinal or respiratory anthrax may develop. Cutaneous anthrax accounts for more than $95 \%$ of all human cases worldwide $(1,2,4,5)$, and mostly seen in the hand, arm, head, or neck, yet this depends on the exposed area (6). The incubation period was noted to be from 1 to 19 days, but usually is between 2 and 9 days $(5,7,8)$. Initiation of appropriate therapy prevents systemic disease but does not alter the evolution of the cutaneous lesion (9). Thus, it is important to accurately diagnose cutaneous anthrax based on the history of the patient and the characteristics of the skin lesions (1).

In this report, we present an illustrated case of cutaneous anthrax acquired in eastern Turkey.

\section{CASE PRESENTATION}

A 56-year-old female patient applied in July 2018 to the infectious diseases outpatient clinic of Ardahan State Hospital with a painless, dark- coloured swelling over her right

\section{Corresponding Author:} Cansu Çimen

E-mail:

cansucmn@yahoo.com

Received: April 20, 2020 Accepted: April28, 2020 Published: April 30, 2020

Suggested citation: Çimen C. Typical Evolution of a Cutaneous Anthrax Lesion. Infect Dis Clin Microbiol 2020; 1: 27-29.

DOI: 10.36519/idem.2020.0007 


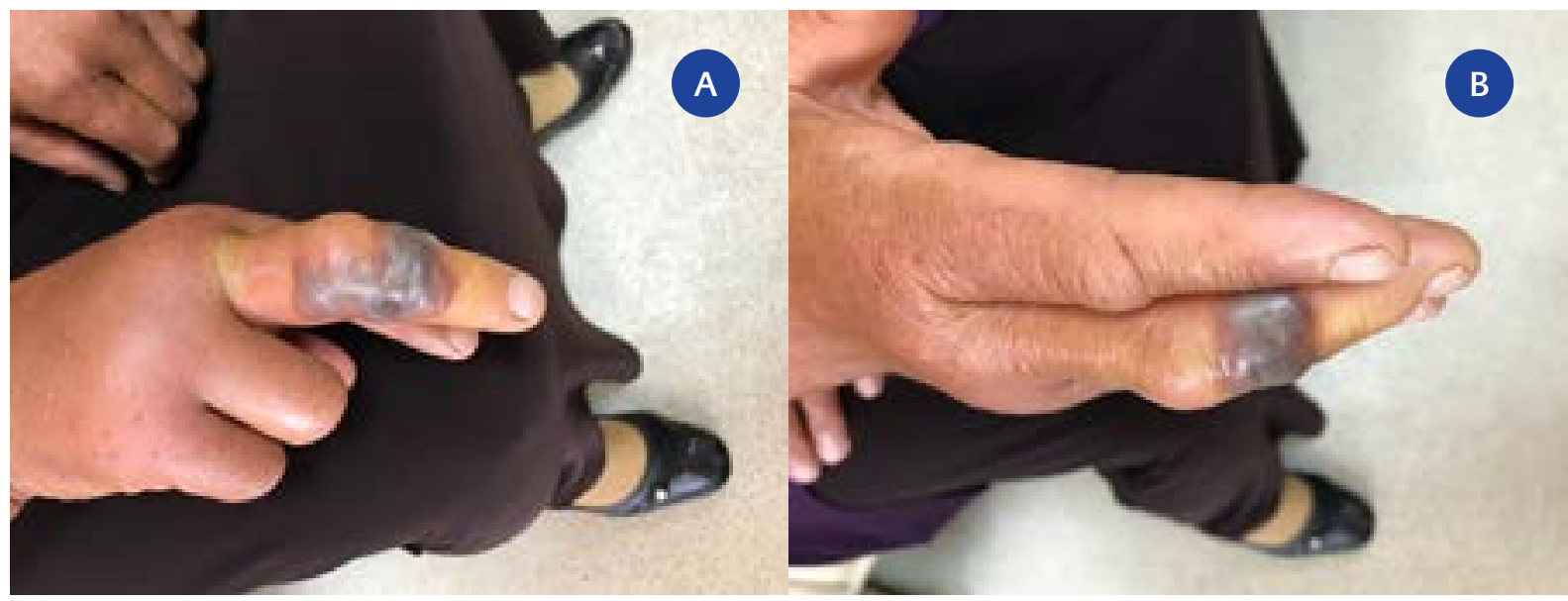

Figure 1. Anthrax lesion at the day of presentation (a) top view, (b) lateral view

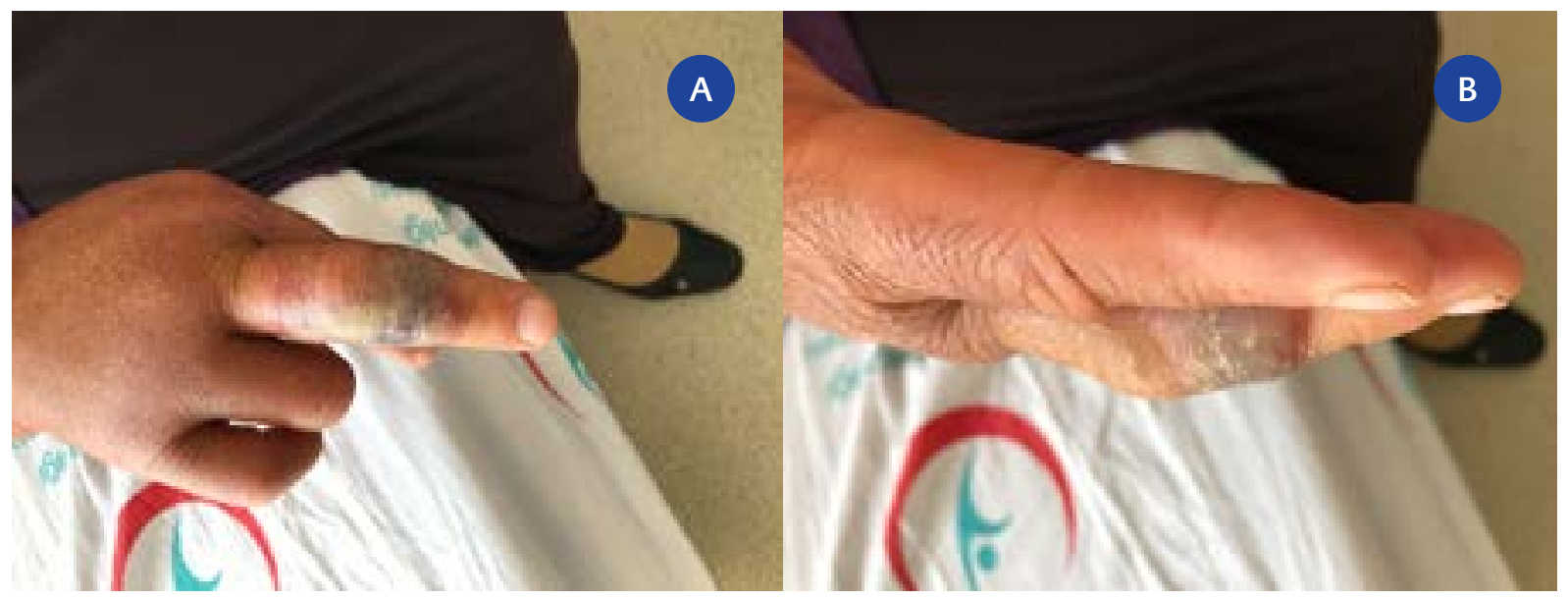

Figure 2. Anthrax lesion on the third day of treatment (a) top view, (b) lateral view

middle finger accompanied by oedema extending to the right hand (Figure $1 \mathrm{a}$ and $1 \mathrm{~b}$ ).

As a farmer, she had noticed the sudden death of several sheep; therefore, she cut their mutton. Three days later, the first lesion appeared. The patient described the initial lesion as a small papule, which expanded over two days and ended with a swollen hand. Despite its violaceous view, the lesion was remarkably painless.

On physical examination; she was afebrile, hemodynamically stable, and there was no neurovascular or orthopaedic injury. She had a tender epitrochlear lymph node of about $3 \mathrm{~cm}$ in diameter. The laboratory examination was regular. After puncture of the lesion, a Gram's stain and culture tests were performed on the aspiration material. Gram-positive, endospore-forming-rods were observed. The patient was started on amoxicillin-clavulanic acid 3gr/day. Three days after the start of the treatment, the swelling had decreased (Figure 2a and 2b).

After ten days of treatment, a typical anthrax eschar with a sharp-edged, black ulcer in the middle of the wound appeared, and the antibiotic was stopped (Figure 3).

\section{DISCUSSION AND CONCLUSION}

This case demonstrates the typical disease course of a cutaneous anthrax lesion. It is helpful to clinicians, as the diagnosis mainly depends on clinical features. A cutaneous anthrax lesion usually begins with itch- 
ing at the site of entry. Subsequently, small, painless papule emerges. This papule quickly enlarges and develops a central vesicle, surrounded by oedema. In a couple of days, the vesicle fluid becomes darker. The wound turns into a necrotic ulcer, followed by a depressed painless eschar. Swollen and painful regional lymph nodes and lymphangitis often accompany this lesion $(5,10,11)$. The differential diagnosis of cutaneous anthrax includes a wide range of infectious diseases: erysipelas, cat-scratch disease, cutaneous plague, ulceroglanduler tularemia, clostridial infection, orf, vaccinia and cowpox, leishmaniasis, ecthyma gangrenosum, blastomycosis, sporotrichosis or herpetic withlow. However, these infections lack the characteristic oedema of anthrax $(1,7)$.

The fatality rate of cutaneous anthrax among humans is $<1 \%$ with adequate treatment, but the rate could increase by up to $20 \%$ in the case of late diagnosis and treatment (4). Oedema associated tracheal compression, severe oedema and shock may develop as a complication $(4,7)$.

Anthrax is an endemic disease, particularly in eastern and southeastern Turkey, where animal hus-

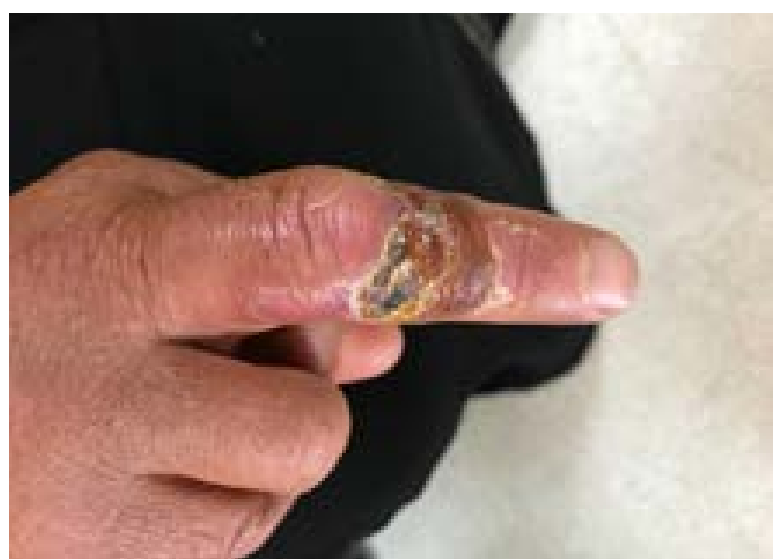

Figure 3. A typical anthrax eschar

bandry and farming are common. The cutaneous form of the disease is diagnosed based on a history of contact with animals or animal products in an endemic region and the presence of a violaceous but painless skin lesion on an edematic background. Considering anthrax in the differential diagnosis is crucial for the prompt and appropriate treatment. This case report may help clinicians to recognize the different stages of the disease in clinical practice.
Peer-review: Externally peer-reviewed

Statement: This case report was presented in Clinical Grand Round of ECCMID 2019, Amsterdam, April 2019
Financial Disclosure: The authors declared that this study has received no financial support.

\section{REFERENCES}

1 Anthrax in Humans and Animals. 4th ed. Turnbull P, editor. Geneva: World Health Organisation; 2008.

2 Dixon TC, Meselson M, Guillemin J, Hanna PC. Anthrax. N Engl. J. Med 1999; 341: 815-826.

3 Hendricks KA, Wright ME, Shadomy SV, Bradley JS, Morrow MG, Pavia AT, et al. Centers for Disease Control and Prevention expert panel meetings on prevention and treatment of anthrax in adults. Emerg Infect Dis 2014; 20: e130687.

4 Shadom SV, Smith TL. Zoonosis update. Anthrax. J Am Vet Med Assoc 2008; 233: 63-72.

5 Doganay M, Metan G, Alp E. A review of cutaneous anthrax and its outcome. J Infect Public Health 2010; 3: 98-105.

6 Kamal SM, Rashid AK, Bakar MA, Ahad MA. Anthrax: An update. Asian Pac J Trop Biomed 2011; 1: 496-501.
7 Kaya A, Tasyaran MA, Erol S, Ozkurt Z, Ozkan B. Anthrax in adults and children: A review of 132 cases in Turkey. Eur J Clin Microbiol. Infect. Dis 2002; 21: 258-261.

8 Abdenour D, Larouze B, Dalichaouche M, Aouati M. Familial Occurrence of Anthrax in Eastern Algeria. J Infect Dis 1987; 155: 1083-4

9 Parlak E, Parlak M. Human Cutaneous Anthrax, the East Anatolian Region of Turkey 2008-2014. Vector-Borne Zoonotic Dis 2016; 16: 42-7.

10 Wenner KA, Kenner JR. Anthrax. Dermatol Clin 2004; 22: 247256.

11 Baykam N, Ergonul O, Ulu A, Eren S, Celikbas A, Eroglu M, et al. Characteristics of cutaneous anthrax in Turkey. J Infect Dev Ctries 2009; 3: 599-603. 